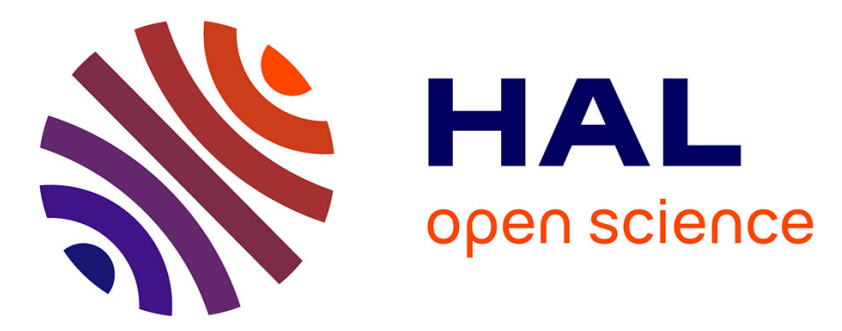

\title{
Outils numériques pour le suivi des patients allergiques. L'exemple du projet MASK-air
}

J. Bousquet, S. Arnavielhe, A. Bedbrook, I. Bossé, W. Czarlewski, P. Demoly, P. Devillier, E. Dupas, Jf. Fontaine, D. Laune, et al.

\section{- To cite this version:}

J. Bousquet, S. Arnavielhe, A. Bedbrook, I. Bossé, W. Czarlewski, et al.. Outils numériques pour le suivi des patients allergiques. L'exemple du projet MASK-air. Revue francaise d'allergologie, 2019, 59, pp.172 - 173. 10.1016/j.reval.2019.02.006 . hal-03486340

\section{HAL Id: hal-03486340 \\ https://hal.science/hal-03486340}

Submitted on 20 Dec 2021

HAL is a multi-disciplinary open access archive for the deposit and dissemination of scientific research documents, whether they are published or not. The documents may come from teaching and research institutions in France or abroad, or from public or private research centers.
L'archive ouverte pluridisciplinaire HAL, est destinée au dépôt et à la diffusion de documents scientifiques de niveau recherche, publiés ou non, émanant des établissements d'enseignement et de recherche français ou étrangers, des laboratoires publics ou privés.

\section{(c) (1) $\$$}

Distributed under a Creative Commons Attribution - NonCommerciall 4.0 International 


\title{
Outils numériques pour le suivi des patients allergiques \\ L'exemple du projet MASK-air
}

\author{
Use of digital tools for follow-up of allergy patients: \\ example of the MASK-air project
}

J Bousquet (1,2), S Arnavielhe (3), A Bedbrook (1), I Bossé (4), W Czarlewski (5), P Demoly (6), P Devillier (7), E Dupas (4), JF Fontaine (8), D Laune (4), N Pham-Thi (9) M Thibaudon

1. MACVIA-France, Contre les MAladies Chroniques pour un Vleillissement Actif en France European Innovation Partnership on Active and Healthy Ageing Reference Site, Montpellier, France.

2. INSERM U 1168, VIMA : Ageing and chronic diseases Epidemiological and public health approaches, Villejuif, Université Versailles St-Quentin-en-Yvelines, UMR-S 1168, Montigny le Bretonneux, France and Euforea, Brussels, Belgium.

4. Kyomed Innov, Montpellier, France.

5. Medical Consulting Czarlewski, Levallois

6. Department of Respiratory Diseases, Montpellier University Hospital, France

7. Laboratoire de Pharmacologie Respiratoire UPRES EA220, Pôle des Maladies Respiratoires, Hôpital Foch, Suresnes Université Versailles Saint-Quentin, France.

8. Reims, France.

9. Institut Pasteur, Paris, France

10. RNSA (Réseau National de Surveillance Aérobiologique), Brussieu, France.

\section{Correspondance}

Pr Jean Bousquet, Fondation FMC VIA-LR, 163 rue Auguste Broussonet, 34000-Montpellier +33-611-42-88-47, jean.bousquet@ orange.fr 
Mots-clés : MASK, ARIA, smartphone, rhinite allergique, traitement

Keywords: MASK, ARIA, smartphone, allergic rhinitis, treatment

\section{Introduction}

La rhinite allergique (RA) est la maladie chronique la plus fréquente au monde. Les recommandations ont amélioré les connaissances sur la RA et ont eu un impact significatif sur leur prise en charge. Cependant, de nombreux patients sont encore insuffisamment contrôlés. La rhinite allergique et son impact sur l'asthme (ARIA) a évolué d'une recommandation utilisant la meilleure approche (GRADE) et des parcours de soins utilisant la technologie mobile pour la RA et la multimorbidité de l'asthme. ARIA semble être proche des besoins du patient, mais des données réelles obtenues en vie réelle et utilisant une application montrent que des progrès importants doivent être réalisés qui devraient changer la prise en charge des maladies allergiques (1).

\section{MASK}

MASK (MACVIA-ARIA Sentinel Network pour la rhinite allergique et la multimorbidité de l'asthme) est un système de technologie de l'information et des communications (TIC). II est centré sur le patient et leur permet d'accéder à une application conviviale sur leur smartphone. C'est l'un des outils de mise en œuvre du Partenariat Européen d'Innovation pour un vieillissement actif et en bonne santé (EIP on AHA), un projet mis en place par la Commission européenne (DG Santé et DG CNECT) (2). MASK est une Bonne Pratique e la DG Santé (Union Européenne). MASK a un nouveau module concernant le sommeil, composante importante de la RA (3) et examine l'impact de la pollution atmosphérique pour l'asthme et la rhinite (4).

Une application gratuite pour téléphones mobiles (appelée MASK-air) a été développée et lancée dans 23 pays (UE, Argentine, Australie, Canada, Brésil, Mexique, Turquie et Suisse) et dans 17 langues (5). Les données sont entièrement anonymisées (6). L'application est fondée sur des échelles visuelles analogiques (EVA) que le patient peut utiliser pour évaluer sa rhinite, sa conjonctivite, contrôler son asthme et sa productivité au travail. L'App a également une liste déroulante comprenant tous les médicaments contre la rhinite et l'asthme disponibles dans le pays du patient. Plus de 26.000 utilisateurs ont été enregistrés. Le traitement (médical et immunothérapie) est inclus dans l'application. Chaque jour, les patients rapportent leurs symptômes (échelle visuelle analogique) et médicaments. Lorsque le patient désire avoir une vision complète de ses symptômes et médications, il peut directement imprimer sur ordinateur une fiche qu'il pourra transmettre à son médecin.

MASK est la première application en allergologie dont la validation méthodologique a été publiée, en particulier la distribution des réponses pour l'échelle visuelle analogique. II s'agit ainsi d'une source de données validée concernant les symptômes journaliers et l'impact sur le travail (2).

\section{Suivi des patients allergiques}


Plusieurs résultats obtenus par MASK permettent d'avoir une approche plus proche des besoins du patient allergique.

Les applications de technologie mobile permettent d'apprécier l'adhérence au traitement. MASK-air a été testé lors d'une étude observationnelle entre janvier 2016 et aout 2017 chez 6.949 utilisateurs. Chez les 1.887 personnes ayant utilisé l'application plus de 7 jours $(27,1 \%)$, moins des $10 \%$ ont été considérés adhérents au traitement (7).

Les études observationnelles en vie réelle sont nécessaires pour apprécier le traitement des maladies allergiques et compléter les essais cliniques randomisés. 2871 utilisateurs de MASK ont rempli le questionnaire pendant 17.091 jours. Les patients utilisent les traitements en fonction de leur contrôle, et contrairement aux recommandations augmentent leur traitement lorsqu'ils ne sont pas contrôlés. En outre, les patients utilisent largement des médicaments en vente libre dans les pharmacies. La prise de décision partagée (SDM) centrée sur le patient devrait être utilisée plus souvent (8). Cette étude pilote a été confirmée lors d'une nouvelle étude chez 10.000 patients.

\section{Implication dans les parcours de soins}

Peu de patients utilisent des recommandations et ont souvent recours à l'auto-médication. Ils utulisent leurs médicaments selon leurs symptômes et ont besoin de traitements d'action rapide (9). Ces notions devront être intégrées dans les parcours de soins innovants fondés sur les études en vraie vie.

\section{References}

1. Bousquet J, Hellings PW, Agache I, Amat F, Annesi-Maesano I, Ansotegui IJ, et al. ARIA Phase 4 (2018): Change management in allergic rhinitis and asthma multimorbidity using mobile technology. J Allergy Clin Immunol. 2018:pii: S0091-6749(18)31359-9. doi: 10.1016/j.jaci.2018.08.049.

2. Bousquet J, Arnavielhe S, Bedbrook A, Bewick M, Laune D, Mathieu-Dupas E, et al. MASK 2017: ARIA digitally-enabled, integrated, person-centred care for rhinitis and asthma multimorbidity using real-world-evidence. Clin Transl Allergy. 2018;8:45.

3. Munoz-Cano R, Ribo P, Araujo G, Giralt E, Sanchez-Lopez J, Valero A. Severity of allergic rhinitis impacts sleep and anxiety: results from a large Spanish cohort. Clin Transl Allergy. 2018;8:23.

4. Bousquet J, Anto JM, Annesi-Maesano I, Dedeu T, Dupas E, Pepin JL, et al. POLLAR: Impact of air POLLution on Asthma and Rhinitis; a European Institute of Innovation and Technology Health (EIT Health) project. Clin Transl Allergy. 2018;8:36.

5. Bousquet J, Agache I, Aliberti MR, Angles R, Annesi-Maesano I, Anto JM, et al. Transfer of innovation on allergic rhinitis and asthma multimorbidity in the elderly (MACVIA-ARIA) - EIP on AHA Twinning Reference Site (GARD research demonstration project). Allergy. 2018;73(1):77-92.

6. Samreth D, Arnavielhe S, Ingenrieth F, Bedbrook A, Onorato GL, Murray R, et al. Geolocation with respect to personal privacy for the Allergy Diary app - a MASK study. World Allergy Organ J. 2018;11(1):15.

7. Menditto E, Costa E, Midao L, Bosnic-Anticevich S, Novellino E, Bialek S, et al. Adherence to treatment in allergic rhinitis using mobile technology. the mask study. Clin Exp Allergy. 2018. 
8. Bousquet J, Devillier P, Arnavielhe S, Bedbrook A, Alexis-Alexandre $G$, van Eerd $M$, et al. Treatment of allergic rhinitis using mobile technology with real-world data: The MASK observational pilot study. Allergy. 2018;73(9):1763-74.

9. Bachert C, Bousquet J, Hellings P. Rapid onset of action and reduced nasal hyperreactivity: new targets in allergic rhinitis management. Clin Transl Allergy. 2018;8:25. 